\title{
Drug information of Aducanumab and its effectiveness for Alzheimer's disease: A clinical review
}

Neel Sureshbhai Raval 1, Sachi Anilkumar Chavda 1, Dhwanil Nileshkumar Mithaiwala 1, SP Srinivas Nayak 2, *, Mohit Buddhdev ${ }^{2}$ and Gunosindhu Chakraborthy ${ }^{3}$

${ }_{1}^{1}$ Parul Institute of Pharmacy and Research, Parul University, Vadodara, Gujarat.

2 Dept. of Pharmacy Practice, Parul Institute of Pharmacy and Research, Parul University, Vadodara, Gujarat.

${ }^{3}$ Prof. and Principal, Parul Institute of Pharmacy and Research, Parul University, Vadodara, Gujarat.

International Journal of Biological and Pharmaceutical Sciences Archive, 2021, 02(02), 072-077

Publication history: Received on 27 October 2021; revised on 20 December 2021; accepted on 22 December 2021

Article DOI: https://doi.org/10.53771/ijbpsa.2021.2.2.0094

\begin{abstract}
Alzheimer's disease (AD) is the main cause of disability in people older than 60 years. More than 1 million cases per year reported in India. AD and other kinds of dementia are more common as individuals become older, affecting 1 in every 14 people over the age of 65 and 1 in every 6 people over the age of 80. ADUHELM (Aducanumab) is a monoclonal antibody, new class of medication needed for treatment of AD. The latest drug used to treat underlying cause of AD and recent studies shown to be efficacious and safe for the AD. Our review emphasizes on the drug information of Aducanumab and its clinical importance in AD.
\end{abstract}

Keywords: Alzheimer's disease; Aducanumab; Drug information; Genetic mutations.

\section{Introduction}

Early onset Alzheimer disease (EOAD) is a neurodegenerative dementing disorder that is relatively rare $(<1 \%$ of all Alzheimer cases). Various genetic mutations of the presenilin 1 (PSEN1) and presenilin 2 (PSEN2) as well as the amyloid precursor protein (APP) gene have been implicated. Mutations of PSEN1 and PSEN2 alter $\gamma$-secretase enzyme that cleaves APP resulting in increase in the relative amount of the more amyloidogenic A $\beta 42$ that is produced. [1] AD is a neurodegenerative condition that causes brain shrinkage and cell death overtime.[2] PSEN2 mutations are reported to be associated with AD of both early onset and variable age onset as well as with other neurodegenerative disorders such as Lewy Body dementia, frontotemporal dementia, Parkinson dementia, and posterior cortical atrophy[2-4].; Patients most often present with an insidious loss of episodic memory followed by a slowly progressive dementia that evolves over years.[3] AD is the most common cause of dementia, which is characterised by a progressive loss of cognitive, behavioural, and social abilities that impairs a person's capacity to operate independently.[1] Dementia is described as a group of symptoms affecting severely to thinking, memory, and social abilities which may interfere with your day-today life.[4] AD establishes around 70\% of all dementia cases. AD is typically an old age disease. The global prevalence of dementia is about 24 million and it's going to increase 4 times by 2050 . AD is expected to cost $\$ 172$ billion a year in health-care costs in the United States alone. The incidence rate of AD after the age of 65 is multiplied by 5 times. Agespecific incidence increases significantly from less than $1 \%$ per year before 65 years of age to $6 \%$ per year after 85 years of age. Prevalence rates increase from $10 \%$ after the age of 65 to $40 \%$ after the age of 85 . Women are somewhat more likely than males to get $\mathrm{AD}$, especially beyond the age of 85 . To date, most validated research results have been narrowed down to yield a model for biological trajectory of $\mathrm{AD}$, where amyloid deposition far precedes clinical symptoms. [5]. Old age and a favourable family history are the two most major risk factors for AD. The prevalence of AD increases with each decade of adult life, reaching $20-40 \%$ of the population over the age of 85 . A positive family history of dementia

\footnotetext{
* Corresponding author: S P Srinivas Nayak, Email id: spnayak843@gmail.com

Assistant Professor, Dept. of Pharmacy Practice, Parul Institute of Pharmacy and Research, Parul University, Vadodara, Gujarat 
suggests a genetic contribution to AD, although autosomal dominant inheritance occurs in only $2 \%$ of patients. Female sex is a risk factor independent of the greater longevity of women, and women who carry an Apo $\varepsilon 4$ allele are more susceptible than are male $\varepsilon 4$ carriers. A history of head trauma with concussion increases the risk for AD.[2] There are four distinct genetic loci linked to inherited AD risk. Amyloid precursor protein, apolipoprotein E epsilon 4, presenilin 1 and presenilin 2 are four genes that have been linked to hereditary vulnerability to AD. Excessive cerebral amyloid beta plaque buildup and consequent neuronal and glial disease in brain regions crucial for sustaining functions like memory and cognition are caused by mutations or polymorphisms in these genes. Interleukin-1 alpha, interleukin-6, interleukin-10, tumour necrosis factor alpha, cystatin C, neprilysin, angiotensin converting enzyme, BACE 1, cathepsin $\mathrm{D}$, and brain derived neurotrophic factor are some of the other genes linked in AD.[6] There have been relatively few reports utilizing $18 \mathrm{~F}$-labelled amyloid beta PET tracers that include clinical implications related to autosomal dominant AD. One study adopted 18F-florbetaben PET imaging in Down syndrome patients, suggesting potential role of amyloid imaging in identifying population at risk of dementia.[6] The major genetic risk factor for AD is apolipoprotein $\varepsilon 4$ (Apo $\varepsilon 4)$. Carrying one E4 allelomorph's increases the risk for AD by 2- to 3-fold, whereas two allelomorph's can increase the risk 16-fold. [2] Microscopic Approach of AD include Neuritic plaques (also known as "senile plaques") are extracellular deposits of the amyloid beta-protein $(A ß)$ that are typical microscopic findings of $A D$. This $A ß$ alone is found as filamentous masses in the more frequent, smaller diffuse plaques. Similar study was conducted on patients with Down syndrome, but with $18 \mathrm{~F}$-florbetapir tracer. $[7,8,9]$

In $\mathrm{AD}$ pathology role of Plaques and tangles are two aberrant formations, are suspected of harming and destroying nerve cells. Plaques are deposits of beta-amyloid, a protein fragment that builds up in the gaps between nerve cells. Tangles are twisted strands of tau, a protein that builds up inside cells.[8] Sign \& Symptoms include Having trouble in remembering what just happened, Inability to plan ahead of time or address issue, Not able to remember the seasons, dates, and time, have a habit of misplacing items, Mood swings and personality shifts, Poor decision-making abilities, Having difficulty having discussions, Finding it difficult to speak/find the appropriate words, Issues with vision, Refusal to participate in social or job activities. An attempt to differentiate Down Syndrome pathology from AD has also been made with $18 \mathrm{~F}$-florbetapir tracer. $[8,10]$

In Clinical Examination, Doctors will ask about any changes in person's quality of life and how it is affecting person's ability to perform daily tasks e.g.: - Managing daily hurdles of life, changing cloths, take medication on time, Performing Daily housework. [9] The doctor will inquire about the patient's current and previous medical problems, medicines, and family history of AD or other memory impairments. He or she will also perform a neurologic exam and examine all current vital signs (blood pressure, heart rate, temperature, pulse rate) (check reflexes and coordination, eye movement, speech, and sensation). Testing for mental health. Memory, problem-solving, concentration, counting, and language abilities are among the exams available. This sort of testing can also be us ed to track AD progression. Neuro psychological evaluations are conducted. Attention, memory, language, planning and reasoning abilities, the capacity to modify behaviour, as well as personality and emotional stability are all assessed in this exam. This sort of testing can also be used to track AD progression. Spine tap is also known as a lumbar puncture, looks for the tau and amyloid proteins that create the plaques and tangles observed in AD patients' brains. Brain Imaging Studies may consist of Computed tomography is a type of brain imaging examination. This scan indicates physical changes in the structure of brain tissue that occur later in $\mathrm{AD}$, such as a reduction in brain size (atrophy), widening of brain tissue indentations, and expansion of the brain's fluid-filled chambers. Magnetic resonance imaging (MRI) is a type of imaging that uses This scan may also reveal signs of brain shrinkage. It can also detect strokes, tumours, fluid accumulation on the brain, and other structural abnormalities that might mimic the symptoms of AD. FMRI stands for functional magnetic resonance imaging (functional MRI). This is a form of MRI that detects variations in blood flow to quantify brain activity in a specific region. Researchers are using this test to understand how the brain changes as AD progresses. It's also being used to test $\mathrm{AD}$ therapies before a person develops symptoms. Positron emission tomography (PET SCAN) is a kind of positron emission tomography. The aberrant brain activity in a person with AD may be shown in this scan. It can also aid in the differentiation of AD from other kinds of dementia. PET for Amyloid. The accumulation of amyloid protein in the brain is seen in this scan. PET with FDG. This image tells how efficiently brain cells utilise glucose. AD is characterised by a decrease in glucose absorption.[10] Complications of AD may include Restlessness \& Agitation, Bowel \& Bladder Problems, Sleeplessness, Mood Swings, Depression, Falls, Infections.[11] In these patients, agitation is widespread and persistent, while psychotic symptoms are less common and only somewhat persistent over time. It's unusual to have a depressed mood with vegetative indications, and it usually doesn't last long. Neuroleptics should be used first for the treatment of psychotic characteristics and agitation.[12] Although there is no cure for AD, one treatment, aducanumab, is the first to indicate that removing amyloid, one of the illness's hallmarks, from the brain can reduce cognitive and functional decline in individuals with early AD. Other treatments can assist persons with AD and their caregivers live better lives by temporarily reducing dementia symptoms and increasing quality of life. There is now a global effort on to find innovative ways to treat, delay, and prevent the sickness from developing.[12]. 
There are mainly 7 stages for AD. Stage 1 include Normal Outward Behaviour, Stage 2 include Very Mild Changes, Stage 3 include Mild Decline, Stage 4 include Moderate Decline, Stage 5 include Moderately Severe Decline, Stage 6 include Severe Decline, Stage 7 include Very Severe Decline.[13]

\section{Management of AD}

Approved anti-AD medications: cholinesterase inhibitors and memantine Cholinesterase inhibitors (donepezil, galantamine, rivastigmine) and the $\mathrm{N}$-methyl-D-aspartate antagonist, memantine, are the only FDA-approved treatments for AD dementia and are recommended mainly in consensus guidelines and practice variables.[13];Until now, the most widely studied combination drug therapy for AD treatment is the concurrent use of memantine and Cholinesterase inhibitors.[14] Optimal quality of care during the early and middle phases of AD requires the identification of triggers for implementation of Cholinesterase inhibitors and memantine, monitoring of patient response to detect triggers for choosing the pharmacological agent and meet the expectation of patient as well as caregivers.[15] Tacrine is used to treat mild to moderate AD symptoms. Tacrine will not cure AD, nor will it slow down the progression of the illness. In certain Alzheimer's sufferers, tacrine, on the other hand, can help them think more clearly.[16] Donepezil is a drug that is used to treat AD-related disorientation (dementia). In individuals with mild to moderately severe AD, donepezil has been found to be well tolerated and to improve cognition and overall function.[17] The cholinesterase inhibitors donepezil, rivastigmine, and galantamine can delay cognitive impairment in patients with mild to moderately severe AD for at least 6 months duration.[18] Memantine is Uncompetitive antagonist of glutamate receptors of the N-methyl-D-aspartate type. Amantadine treatment resulted in a significant improvement in mental state in three individuals whose autopsy later revealed AD. During the therapy, two patients exhibited a significant improvement. They were able to say a few intelligible sentences when their silent, immobile statuses were reversed.[19]Evidence-based guidelines outlining pharmacotherapeutic strategies can be systematically implemented to optimize outcomes for patients in different stages of AD.[20]

\section{Drug information of aducanumab}

Aducanumab (ADUHELM) is a monoclonal antibody indicated in the treatment of AD. The recommended dose of ADUHELM is $10 \mathrm{mg} / \mathrm{kg}$ IV over 1 hour every 4 weeks and atleast21 days apart. ADUHELM is a human, immunoglobulin gamma 1(IgG1) monoclonal antibody which reduces aggregated soluble and insoluble amyloid beta plaques in patient with AD.

\subsection{Adverse drug reaction}

Dermatologic ADR include Urticaria Immunologic ADR include Angioedema Neurologic ADR include Magnetic resonance imaging of brain abnormal, Amyloid related imaging abnormalities-edema (35\%), Amyloid-related imaging abnormalities-hemosiderin deposition (35\%) Side effects may include headache, fall, confusion and diarrhea.

\subsection{Drug interaction}

No interactions were found.

\subsection{Use in pregnancy and lactation}

There are no adequate data on the developmental risk associated with the use of ADUHELM in pregnant women. There are no data on the presence in human milk, effects of on the breastfed infant, or the effects on milk production. Safety and effectiveness in pediatric patients have not been established.

\subsection{Dose adjustment in organ impairment}

No dosage adjustment is needed for patients with renal and hepatic impairment. Also, no specific recommendations are available for geriatric patients. If signs or symptoms of hypersensitivity type of reaction occurs, then promptly discontinue the infusion.

\subsection{Contraindications}

Specific contraindications have not been determined. 


\subsection{Precautions}

Neurologic: Use may cause amyloid-related imaging abnormalities-edema (ARIA-E), which may be observed on MRI as brain edema or sulcal effusions, and amyloid-related imaging abnormalities-hemosiderin deposition (ARIA-H), which includes microhemorrhage and superficial siderosis. Obtain recent (within 1 year) brain MRI prior to initiation treatment; monitoring is recommended.

Neurologic: The safety of use in patients with any pre-treatment localized superficial siderosis, 10 or more brain microhemorrhages, and/or with a brain hemorrhage greater than $1 \mathrm{~cm}$ within 1 year of treatment initiation has not been established.

Immunologic: Angioedema and urticaria has been reported; treatment and prompt discontinuation of therapy may be necessary.

\subsection{Pharmacokinetics}

Absorption by intravenous route $10 \mathrm{mg} / \mathrm{kg}$ dose of aducanumab reached a $\mathrm{C}$ max of $182.7 \mu \mathrm{g} / \mathrm{mL}$, with a T max of 3.0 hours, and an AUCinf of $31,400 \mathrm{~h}^{*} \mathrm{\mu g} / \mathrm{mL}$. The volume of distribution of aducanumab is $9.63 \mathrm{~L}$. Metabolism of Aducanumab is expected to be broken down into smaller oligopeptides and amino acids. Route of elimination of Monoclonal IgG is predominantly eliminated by catabolism to individual amino acids that are either recycled in the body or metabolized for energy. The terminal half-life of aducanumab is 24.8 days. A $10 \mathrm{mg} / \mathrm{kg}$ intravenous dose of aducanumab has a clearance of $0.39 \mathrm{~mL} / \mathrm{h} / \mathrm{kg}$.

\subsection{Effectiveness of aducanumab}

Aducanumab dosed at $30 \mathrm{mg} / \mathrm{kg}$ was efficacious and well tolerated in the treatment of AD without any potential adverse effects.[21];Aducanumab, is a newer treatment of immunotherapy that can decrease the pathological damage of AD and reverse or slow down the declining of cognitive function for same. [22] A study on effectiveness of aducanumab was done and result shows that it penetrates the brain and anti-beta amyloid concentration decreases with time and dosedependent manner. After around 15 months of treatment with aducanumab, data shows that $3 \mathrm{mg} / \mathrm{kg}, 6 \mathrm{mg} / \mathrm{kg}$ and 10 $\mathrm{mg} / \mathrm{kg}$ doses show the significant reduction of beta amyloid plaque in patients with AD. They have also conducted a preclinical study on rat and administered $30 \mathrm{mg} / \mathrm{kg}$ dose via intra-peritoneal route which confronts that $30 \mathrm{mg} / \mathrm{kg}$ dose is required for diffusion and closely bound with beta amyloid plaque. In this study, they found some adverse drug reaction like headache, urinary tract infection and upper respiratory tract infection which are related to amyloid-related imaging abnormalities (ARIA).[23] In a Phase I clinical trial, 166 patients with mild prophylaxis or mild symptoms were given $1,3,6$, or $10 \mathrm{mg} / \mathrm{kg}$ doses of Aducanumab every 4 weeks, and after 54 weeks of treatment, the 3 and $10 \mathrm{mg} / \mathrm{kg}$ doses of the drug significantly reduced $\mathrm{Ab}$ content in the brain and improved cognitive ability, with the higher dose being more pronounced, compared to placebo. Although the findings of the $6 \mathrm{mg} / \mathrm{kg}$ dose group did not differ from those of the 3 $\mathrm{mg} / \mathrm{kg}$ and $10 \mathrm{mg} / \mathrm{kg}$ dose groups, the dose of $6 \mathrm{mg} / \mathrm{kg}$ was able to dramatically lower Ab levels in the brain, slowing the patient's mental decline. [23]. Aducanumab can also be used with scanning ultrasound for not only improves cognitive functions but also reduce the beta amyloid plaque load. They found that aducanumab analogue or scanning ultrasound alone can reduce beta amyloid plaque load, but combination therapy has an additional effect on improving cognitive function which is confirmed by active place avoidance (APA) test. 24] All the other patient's, who received 60 $\mathrm{mg} / \mathrm{kg}$ aducanumab has developed systemic adverse drug reaction, which are symptomatic amyloid-related imaging abnormalities (SARI). These were also completely reversed by 2-3 months.[22] Amyloid protein clumps in the brain (plaques) are a neuropathological feature of Alzheimer's disease and widely assumed to trigger a cascade of changes that cause cognitive decline. Aducanumab is a monoclonal antibody that removes amyloid plaques[24]

The study was conducted to found out long-term effects of aducanumab, which shows positive outcome with almost less or no potent adverse effect, which makes this molecule a ray of hope for the patients of AD. Some see aducanumab as proof of concept for the amyloid cascade theory, justifying decades of unsuccessful research costing billions of pounds and exposing thousands of participants to the side effects of experimental treatments. Others fear it will simply encourage futile investment in anti-amyloid therapies, diverting funds away from effective prevention measures such as improving physical activity or reducing hypertension [25]

\section{Conclusion}

$\mathrm{AD}$ is the leading cause of impairment among those over the age of 60 . In India, about a million cases are recorded each year. Is a monoclonal antibody, which is a novel type of medicine used to treat AD. Aducanumab a the first ever disease modifying drug and new medication that treats the fundamental cause of AD, however it has some controversies on its 
efficacy and approval but has been demonstrated in recent trials to be effective and safe for Alzheimer's patients. Genetic counseling may be required in some circumstances, and all patients and care partners require education and support. Building this infrastructure for the appropriate use of aducanumab will require time, resources, and creative planning. Appropriate use of aducanumab requires a commitment to patient-centered care and best-practices for the safe delivery of this new treatment.

\section{Compliance with ethical standards}

\section{Acknowledgments}

We would like to thank our Principal Sir, staff and our friends for supporting us in writing this review article, institute's library, and non-teaching staff for their support.

\section{Disclosure of conflict of interest}

No conflict of interest existed between authors.

\section{References}

[1] Ryman DC, Acosta-Baena N, Aisen PS, et al. Symptom onset in autosomal dominant Alzheimer disease: a systematic review and meta-analysis. Neurology. 2014; 83:253-260

[2] Tremolizzo L, Susani E, Mapelli C, et al. First report of PSEN2 mutation presenting as posterior cortical atrophy. Alzheimer Dis Assoc Disord. 2015;29:249-251.

[3] Cai Y, An SSA, Kim S. Mutations in presenilin 2 and its implications in Alzheimer's disease and other dementiaassociated disorders. Clin Interv Aging. 2015;10:1163-1172.

[4] Youn YC, Bagyinszky E, Kim H, et al. Probable novel PSEN2 Val214Leu mutation in Alzheimer's disease supported by structural prediction. BMC Neurol. 2014;14:105-110

[5] Jack CR, Jr, Knopman DS, Jagust WJ, Shaw LM, Aisen PS, Weiner MW, et al. Hypothetical model of dynamic biomarkers of the Alzheimer's pathological cascade. Lancet Neurol. 2010;9:119-128.

[6] Jennings D, Seibyl J, Sabbagh M, Lai F, Hopkins W, Bullich S, et al. Age dependence of brain $\beta$-amyloid deposition in Down syndrome: An [18F] florbetaben PET study. Neurology. 2015;84:500-507.

[7] Kumar A, Sidhu J, Goyal A, Tsao JW. Alzheimer disease.

[8] Dhingra D, Parle M, Kulkarni SK. Genetic basis of Alzheimer's disease. Indian Journal of Pharmaceutical Sciences. 2005; 67(4): 409.

[9] Sabbagh MN, Chen K, Rogers J, Fleisher AS, Liebsack C, Bandy D, et al. Florbetapir PET, FDG PET, and MRI in Down syndrome individuals with and without Alzheimer's dementia. Alzheimers Dement. 2015;11:994-1004

[10] Matthews DC, Lukic AS, Andrews RD, Marendic B, Brewer J, Rissman RA, et al. Dissociation of Down syndrome and Alzheimer's disease effects with imaging. Alzheimers Dement. 2016;2:69-81

[11] Devanand DP. Behavioral complications and their treatment in Alzheimer's disease. Geriatrics (Basel, Switzerland). 1 Sep 1997; 52: S37-9.

[12] Atri A. The Alzheimer's disease clinical spectrum: Diagnosis and management. Medical Clinics. 1 Mar 2019; 103(2): 263-93.

[13] Kabir M, Uddin M, Mamun AA, Jeandet P, Aleya L, Mansouri RA, Ashraf GM, Mathew B, Bin-Jumah MN, Abdel-Daim MM. Combination drug therapy for the management of Alzheimer's disease. International journal of molecular sciences. 21 Jan 2020; 21(9): 3272.

[14] Farlow MR, Cummings JL. Effective pharmacologic management of Alzheimer's disease. The American journal of medicine. 1 May 2007; 120(5): 388-97.

[15] Burns A, Rossor M, Hecker J, Gauthier S, Petit H, Möller H, -J, Rogers S, L, Friedhoff L, T: The Effects of Donepezil in Alzheimer's Disease - Results from a Multinational Trial<sup >1</sup>. Dement Geriatr Cogn Disord. 1999; 10: 237-244. 
[16] Takeda A, Loveman E, Clegg A, Kirby J, Picot J, Payne E, Green C. A systematic review of the clinical effectiveness of donepezil, rivastigmine and galantamine on cognition, quality of life and adverse events in Alzheimer's disease. International Journal of Geriatric Psychiatry: A journal of the psychiatry of late life and allied sciences. Jan 2006; 21(1): 17-28.

[17] Erkulwater SA, Pillai RE. Amantadine and the end-stage dementia of Alzheimer's type. Southern medical journal. 1 May 1989; 82(5): 550-4.

[18] Farlow MR, Cummings JL. Effective pharmacologic management of Alzheimer's disease. The American journal of medicine. 1 May 2007; 120(5): 388-97.

[19] Ferrero J, Williams L, Stella H, Leitermann K, Mikulskis A, O'Gorman J, Sevigny J. First-in-human, double-blind, placebo-controlled, single-dose escalation study of aducanumab (BIIB037) in mild-to-moderate Alzheimer's disease. Alzheimer's \& Dementia: Translational Research \& Clinical Interventions. 1 Sep 2016; 2(3): 169-76.

[20] Mo JJ, Li JY, Yang Z, Liu Z, Feng JS. Efficacy and safety of anti-amyloid- $\beta$ immunotherapy for Alzheimer's disease: a systematic review and network meta-analysis. Annals of clinical and translational neurology. Dec 2017; 4(12): 931-42.

[21] Sevigny J, Chiao P, Bussière T, Weinreb PH, Williams L, Maier M, Dunstan R, Salloway S, Chen T, Ling Y, O'Gorman $J$. The antibody aducanumab reduces $A \beta$ plaques in Alzheimer's disease. Nature. Sep 2016; 537(7618): 50-6.

[22] Leinenga G, Koh WK, Götz J. A comparative study of the effects of Aducanumab and scanning ultrasound on amyloid plaques and behavior in the APP23 mouse model of Alzheimer disease. Alzheimer's research \& therapy. Dec 2021; 13(1): 1-4.

[23] Herring WL, Gould IG, Fillit H, Lindgren P, Forrestal F, Thompson R, Pemberton-Ross P. Predicted Lifetime Health Outcomes for Aducanumab in Patients with Early Alzheimer's Disease. Neurology and Therapy. 23 Aug 2021; 122.

[24] Sevigny J, Chiao P, Bussière T, et al. . The antibody aducanumab reduces A $\beta$ plaques in Alzheimer's disease. Nature 2016;537:50-6. 10.1038/nature19323.

[25] World Health Organization. Risk reduction of cognitive decline and dementia: WHO guidelines. 2019. 